Submitted to J. Chem. Phys. (2002)

\title{
Cell Multipole Method for Molecular Simulations in Bulk and Confined Systems
}

\author{
Jie Zheng, ${ }^{a, b}$ Ramkumar Balasundaram, ${ }^{a}$ Stevin H.Gehrke, ${ }^{a}$ \\ Grant S. Heffelfinger, ${ }^{c}$ William A. Goddard III, ${ }^{* d}$ and Shaoyi Jiang ${ }^{* b}$ \\ a Department of Chemical Engineering, Kansas State University, Manhattan, KS 66506 \\ b Department of Chemical Engineering, University of Washington, Seattle, WA 98195 \\ c Computational Biology and Materials Technology Department, Sandia National \\ Laboratories, Albuquerque, NM 87185 \\ d Materials and Process Simulation Center, Beckman Institute, Division of Chemistry \\ and Chemical Engineering, California Institute of Technology, Pasadena, CA 91125
}

\begin{abstract}
One of the bottlenecks in molecular simulations is to treat large systems involving electrostatic interactions. Computational time in conventional molecular simulation methods scales with $\mathrm{O}\left(\mathrm{N}^{2}\right)$, where $\mathrm{N}$ is the number of atoms. With the emergence of the cell multipole method (CMM) and massively parallel supercomputers, simulations of 10million atoms have been performed. In this work, the optimal hierarchy cell level and the algorithm for Taylor expansion were recommended for fast and accurate molecular dynamics (MD) simulations of three-dimensional (3D) systems. CMM was then extended to treat quasi-two-dimensional (2D) systems, which is very important for condensed matter physics problems. In addition, CMM was applied to grand canonical ensemble Monte Carlo (GCMC) simulations for both 3D and 2D systems. Under the optimal conditions, our results show that computational time is approximately linear with $\mathrm{N}$ for large systems, average error in total potential energy is less than $\sim 1 \%$, and RMS force is about 0.015 for $3 D$ and $2 D$ systems when compared with the Ewald summation.
\end{abstract}

\footnotetext{
* Author to whom correspondence should be addressed
} 


\section{DISCLAIMER}

This report was prepared as an account of work sponsored by an agency of the United States Government. Neither the United States Government nor any agency thereof, nor any of their employees, makes any warranty, express or implied, or assumes any legal liability or responsibility for the eccuracy, completeness, or usefulness of any information, apparatus, product, or process disclosed, or represents that its use would not infringe privately owned rights. Reference herein to any specific commercial product, process, or service by trade name, trademark, manufacturer, or otherwise does not necessarily constitute or imply its endorsement, recommendation, or favoring by the United States Government or any agency thereof. The views and opinions of authors expressed herein do not necessarily state or reflect those of the United States Government or any agency thereof. 


\section{DISCLAIMER}

Portions of this document may be illegible in electronic image products. Images are produced from the best available original document. 


\section{INTRODUCTION}

The accurate treatment of the long-range Coulombic interactions for large systems is one of the most challenging tasks in computer simulations of charged particles. For a three-dimensional periodic system, the Ewald summation method (EW3D) has been widely used to handle the long-range electrostatic interactions between charged particles. However, the Ewald summation method is computationally very expensive since its complexity is $\mathrm{O}\left(\mathrm{N}^{1.5}\right)$ in a $\mathrm{N}$-particle system. A common approach is to truncate the interactions at a certain cutoff distance. This reduces the operation count to $O(N)$, but significantly scarifies the accuracy, particularly for long-range Coulombic interactions. ${ }^{[1]}$ Recently, much effort has been devoted to improving the efficiency of the Ewald summation method and developing alternative methods for large systems, such as the particle-particle and particle-mesh method (PPPM) $)^{[4,5]}$ and the cell multipole method ${ }^{[1,2,3]}$ (CMM). Both PPPM and CMM are more efficient than the Ewald summation. However, CMM is much more efficient than PPPM for large systems involving 100,000 or more atoms. Furthermore, CMM is well suited to massively parallel supercomputers due to its hierarchy tree structure. In particular, its multipole-based and hierarchical cell structures are well suited to calculating the long-range interactions in large systems. The cost reduces from $\mathrm{O}\left(\mathrm{N}^{2}\right)$ to $\mathrm{O}(\mathrm{N} \log \mathrm{N})$. With growing interest in surface and interfacial systems, it is desirable to apply CMM to quasi-two-dimensional (2D) systems, where periodicity exists in only two dimensions.

In this work, the optimal hierarchy cell (e.g. level 2 or 3 ) and the algorithm for Taylor expansion (e.g., complex or simple downward) were recommended for fast and accurate molecular dynamics (MD) simulations of three-dimensional (3D) systems. CMM was extended to MD simulations for 2D systems and grand canonical ensemble Monto Carlo (GCMC) simulations for both 3D and 2D systems. These methods were tested on pure, binary, and ternary systems. The next section describes the basic ideas of CMM and reduced cell multipole method (RCMM) for periodic systems in MD simulations, and the extension of CMM to confined systems and to GCMC simulations. In the third section, various simulation results of pure and mixed components (e.g., water, 
methane, and dendrimers) in bulk and confined systems by MD and GCMC simulations are given. Conclusions are presented in the final section.

\section{METHODOLOGY}

\section{A. Multipole Approximation}

The key feature of CMM is that by dividing a system into cubic cells and using multipoles (i.e., charges, dipoles, and quadruoles) to represent the cells, a large number of atoms that lead to $\mathrm{N}^{2}$ computations for calculating long-range interactions in the far-field are replaced by these multipole moments ${ }^{[6,7]}$. This replacement reduces computational time to $\mathrm{O}(\mathrm{N} \log N)$, but does not lose accuracy. In order to maintain accuracy, the nearby interactions are computed explicitly while the distant interactions are evaluated by multipole expansions.

$\mathrm{CMM}$ is used for any inverse power-law interaction potential ${ }^{[2]}$ as $V=\sum_{i>j} q_{i} q_{j} /\left|\vec{r}_{i}-\vec{r}_{j}\right|^{p}$, where $p=1$ for Coulombic, $p=6$ for Lennard-Jones (LJ) dispersive, and $p=12$ for $\mathrm{LJ}$ repulsive interactions. CMM can be divided into four parts [1].

- Cell decomposition. The simulation system is decomposed into a hierarchy of cells like a tree structure. The root of the tree is the original system that is defined as level 0 while the leaf of the tree is at the deepest level. The effect of each cell will be represented by multipole expansions.

- Multipole expansion. The multipole moments for all the cells at the deepest level are first determined. Then, the multipoles at the higher level are calculated by combining the corresponding multipole moments of eight children cells at the lower level. This process starts from the deepest level, then moves upward to the root level (level 0 ).

- Far-field multipole Taylor expansion. The multipole moments in the far-field are the same for each atom in a given leaf cell. To calculate force and energy of each atom within a leaf cell, it needs to sum all of the Taylor expansion coefficients by expanding the multipole moments from all distant cells in the far-field to the center of the given cell instead of to each atom. The simple downward algorithm involves the Taylor series V(i), which are calculated by directly summing all the far-field 
contributions. An alternative efficient method for calculating the Taylor expansion coefficients is to share the Taylor coefficients of the parent cell to its children cells, and it is called a complex downward algorithm.

- Near-field and far-field computations. For a given leaf cell, it has 27 neighbor cells including itself and the interactions in this near-field are computed explicitly. The remaining far-field interactions are evaluated by multipole moment expansions.

RCMM is a relatively simple way of extending CMM to periodic systems. The most difficult problem with infinite crystals is to compute the Coulombic interactions, $V_{i}=\sum_{i} / q_{j} / \vec{r}_{i j}$, which are conditionally convergent ${ }^{[3]}$. The main idea of RCMM is that the original unit cell (100 or 10 million atoms) is replaced by a reduced cell containing a small number of randomly positioned fictitious charges (e.g. 35) since the difference between the reduced cell and the original cell is at the $\mathrm{K}^{\text {th }}$ multipole moments which fall off very fast as $1 / r^{K+1}$. In RCMM, the Coulombic interactions are divided into two terms

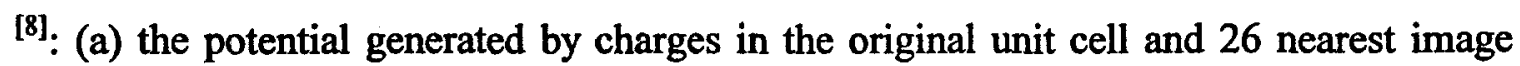
neighbor cells, and (b) the potential due to the fictitious charges in the remaining $\infty-27$ cells. The first term (a) is calculated with CMM, and the second term (b) involves Ewald summation over $\mathbf{P}$ fictitious atoms.

\section{B. Extension to 2D systems}

The RCMM method can be used to evaluate potential energy and force in bulk systems where periodic boundary conditions are used in three dimensions. However, one often encounters systems that are finite in one of the dimensions, such as adsorption and diffusion in slit pores. It is desirable to have a fast and accurate method to treat quasi-2D systems. One of the solutions is to use the EW3D for the quasi-2D systems by introducing a large vacuum gap on the top of the slab. The height of vacuum needs to be adjusted so that it avoids artificial effects in this direction. The method is also computationally very slow. Various other approaches have been developed to deal with the quasi-2D systems. Parry ${ }^{[9]}$ adapted the Ewald transformation to a laminar and semiinfinite system. Heyes and co-workers rederived surface formulas for point-charge and 
point-dipole $^{[10-16]}$ interactions to calculate potential energy and force in molecular simulations. These two methods are found to be most accurate, but the direct use of a Ewald 2D formula is known to be computationally very expensive. ${ }^{[24]}$ Lekner ${ }^{[17]}$ developed a simple surface potential formula cast entirely as a Fourier space series. However, this method was devised originally for square systems. Although the scheme has been extended to rectangular systems, its applicability is still limited ${ }^{[22]}$. Hautman and $\mathrm{Klein}^{[18]}(\mathrm{HK})$ developed a novel expansion procedure, in which the $1 / r$ interaction was decomposed into the in-plane and out-of-plane components in real space. But the HK method was only applied for the systems where the distribution of charge ions in the $z$ direction is small. ${ }^{[22]}$

In this work, a generalized quasi-2D CMM (CMM2D) method was developed by combining the Heyes method with RCMM for confined systems. The methodology of CMM and RCMM described in the previous section still can be applied to the quasi-2D systems. But, the periodic boundary condition is eliminated in the $\mathrm{z}$ direction, and the conventional EW3D summation used in RCMM to deal with the fictitious charges was replaced by the Heyes method. In the Heyes method, the surface lattice is constructed from layers of unit cells infinite in the $\mathrm{x}$ and $\mathrm{y}$ directions. The $2 \mathrm{D}$ real-space lattice vector $\vec{n}$ is denoted as $\vec{n}=\left(n_{x} L, n_{y} L, L\right)$ with $\mathrm{n}_{\mathrm{x}}, \mathrm{n}_{\mathrm{y}}$ integers and reciprocal lattice vector $\vec{k}=2 \pi \vec{n} / L$. The real-space part of the potential energy is shown to be

$$
V_{\text {real }}=\frac{1}{2} \sum_{i=1}^{N} \sum_{j=1}^{N} \sum_{\mid \vec{n}=0}^{\infty}{ }^{\prime} q_{,} q_{j} \frac{\operatorname{erfc}\left(\eta \overrightarrow{r_{i j}}+\vec{n} \mid\right)}{\left|\vec{r}_{i j}+\vec{n}\right|}
$$

where the adjustable parameter $\eta$ is an arbitrary inverse-length parameter; the $\operatorname{erfc}(x)$ $\left(\operatorname{erfc}(x)=(2 / \sqrt{\pi})\left[\exp \left(-t^{2}\right) d t\right)\right.$ is a complementary error function, which falls to 0 with increasing $\mathrm{x}$. The value of $\eta$ determines relative emphasis given to the real- and reciprocal-space terms. If $\eta$ increases, the real-space terms become less important and the reciprocal-space terms become more important due to the erfc(x) function. The prime 
indicates that the case $i=j$ must be omitted for $|\vec{n}|=0$ since a particle cannot interact with itself. The reciprocal-space contribution to the potential energy is shown to be

$$
V_{\text {reciprocal }}=\frac{1}{2} \frac{\pi}{A} \sum_{i=1}^{N} \sum_{j=1}^{N} \sum_{\vec{K}} q_{i} q_{j} F\left(\eta, \vec{K}, r_{z i j}\right) \operatorname{Re}\left[\exp \left(\mathrm{i} \overrightarrow{\mathrm{K}} \cdot \overrightarrow{r_{\mathrm{ij}}}\right)\right]
$$

where $r_{\mathrm{zij}}$ is the $\mathrm{Z}$ component of $\vec{r}_{i j}$. The in-plane area of the unit cell, $\mathrm{A}$, is equal to $A=\left|\vec{L}_{x} \cdot \vec{L}_{y}\right| \cdot$ Re denotes the real part of a complex quantity.

$$
\begin{aligned}
& F\left(\eta, \vec{K} \neq 0, r_{z i j}\right)=\frac{\left\{\exp \left(K r_{z i j}\right) \operatorname{erfc}\left(K / 2 \eta+r_{z i j} \eta\right)+\exp \left(-K r_{z i j}\right) \operatorname{erfc}\left(K / 2 \eta-r_{z i j} \eta\right)\right\}}{K} \\
& \text { If } \vec{K} \neq 0 \\
& F\left(\eta, \vec{K}=0, r_{z i j}\right)=-2\left\{r_{z i j} \operatorname{erf}\left(r_{z i j} \eta\right)+\frac{\exp \left[-\left(r_{z i j} \eta\right)^{2}\right]}{\eta \sqrt{\pi}}\right\} \quad \text { If } \vec{K}=0
\end{aligned}
$$

The existence of a distinct nonzero term when $\vec{K}=0$ is one of the features of surface formula that distinguishes it from the corresponding bulk Ewald expression ${ }^{[16]}$, for which the comparable term is equal to zero for an overall charge neutral system. The self-energy term should be subtracted from the total potential energy as for the bulk case.

$$
V_{\text {self }}=-\frac{\eta}{\sqrt{\pi}} \sum_{i=1}^{N} q_{i}^{2}
$$

The final result for Coulombic interactions in quasi-2D systems is

$$
V=V_{\text {real }}+V_{\text {reciprocal }}-V_{\text {self }} \text {. }
$$

In order to check our CMM2D simulation results, we applied the EW3D technique to quasi-2D systems by adding a large vacuum space on the top of the slab. The inclusion of the vacuum space into the unit cell was done to avoid an artificial influence from periodic images in the $\mathrm{z}$ direction. ${ }^{[20,21]} \mathrm{Spohr}{ }^{[21]}$ compared the results from this approach with those from the two-dimensional Ewald summation (EW2D) method which was first introduced by Parry ${ }^{[9]}$ and later independently rederived by Heyes, Barber, and Clarke ${ }^{[10]}$. 
It was concluded that results for this approach converged to those of EW2D when the vacuum height was large in the $\mathrm{z}$ direction. By increasing the height of the vacuum space in the $\mathrm{z}$ direction, the slab with the vacuum space on its top can be modeled as a strict slab system. The real-space lattice sum decreases quickly because $1 /\left|\overrightarrow{r_{i j}}+\vec{n}\right|$ falls off very fast in the $\mathrm{z}$ direction. The reciprocal vector $\vec{K}=2 \pi \vec{n} / L$ also leads to a decrease in both $\exp \left(-K^{2} / 4 \eta^{2}\right)$ and $4 \pi^{2} / K^{2}$ terms due to the large vacuum in the $\mathrm{z}$ direction. Thus, interactions between the original simulation box and its image cells in the $\mathrm{z}$ direction with large vacuum height for a bulk system were small. Potential energy will converge within a range of the vacuum height.

\section{CMM in GCMC simulations}

In grand canonical ensemble MC (GCMC) simulations, chemical potential is fixed while the number of particles fluctuates. The simulations are carried out at constant $\mu, \mathrm{V}, \mathrm{T}$ (chemical potential, volume and temperature). In GCMC simulations, there are three different types of trials:

(a) a molecule is moved

(b) a molecule is destroyed

(c) a molecule is created at a random position

Each simulation step consists of one of the three attempts described above. For a move attempt, the probability of a movement attempt being accepted is

$$
P_{\text {move }}^{\text {occ }}=\min [1, \exp (-\beta \Delta U(r))]
$$

The probability of creation attempt being accepted is

$$
P_{\text {creation }}^{\text {acc }}=\min \left[1, \exp (-\beta \Delta U(r))+B_{1}-\ln \left(N_{1}+1\right)\right]
$$

The probability of deletion being accepted is

$$
P_{\text {delete }}^{\text {acc }}=\min \left[1, \exp (-\beta \Delta U(r))+\ln N_{i}-B_{i}\right]
$$

Here, $\Delta U(r)$ is the change in configuration energy; $N_{i}$ is the number of molecules; $V$ is the volume; and $\mathrm{B}$ is defined as

$$
B_{i}=\beta \mu_{i}+\ln V
$$


where $\mu_{i}$ is the configurational chemical potential of component $i$.

For a creation attempt, when a new molecule is inserted to the original simulation box, it is assigned to a specific cell based on its coordinates. Then, both the molecule coordinates and the cell number for the inserted molecule are labeled. The new trial configuration is updated in the original simulation box and copied to the image cells. From the hierarchy tree structure, it is easy to identify near, far and $\infty-27$ reduced cells for the labeled cell. Finally, potential energy between this molecule in the labeled cell and the updated system including the inserted molecule and its image cells is calculated using the methods discussed above. The same procedure is used for deletion and movement attempts. The GCMC-CMM program was tested for a binary system including dendrimer and water.

\section{RESULTS AND DISCUSSION}

In this work, applications of the CMM method to MD and GCMC simulations of bulk and confined systems will be discussed. The computational speed and accuracy of the CMM method for evaluating potential energy and force were compared with those from the Ewald summation, minimum image, and Massively Parallel Simulation (MPSim). The calculations with the Ewald summation and the minimum image (half-box cutoff) were achieved using the commercial software package Cerius ${ }^{2}$. MPSim ${ }^{[1]}$ is a parallel simulation program with the CMM method developed by Professor Goddard III's group at California Institute of Technology. All cases were tested on a $192 \mathrm{MHz}$ Silicon Graphics $\mathrm{O} 2$ workstation. All structures were built using Cerius ${ }^{2}$ from Accelrys Inc.

\section{A. MD simulations of bulk systems}

Tests were first performed on the model of water. The $\mathrm{LJ}$ and Coulombic parameters of water for $\quad \sigma_{H}=2.886 \AA, \quad \varepsilon_{H} / k=22.144 \mathrm{~K}, \quad \mathrm{q}_{\mathrm{H}}=0.41 \mathrm{ev}$, $\sigma_{o}=3.50 \AA, \varepsilon_{0} / k=30.196 \mathrm{~K}$, and $\mathrm{q}_{\mathrm{o}}=-0.82 \mathrm{ev}$. The system consists of 512 water molecules with a dimension of $36 \times 36 \times 36 \AA$. Results are listed in Table 1 and are 
shown in Figure 1. Tables 2 and 3 present the energy decompositions for van der Waals (vdw) and Coulombic interactions.

Regarding the level of CMM, as compared with level 1, computational time was reduced by 6.3 times at level 2 for both the simple and complex downward algorithms, and by 4.3 and 5.7 times at level 3 for the simple and complex downward algorithms, respectively. Most computational time at level 1 was spent in calculating the near-field interactions that amount to $8 \times 27 \times 192^{2}=7,962,624$ pairwise interactions. However, at levels 2 and 3 , the total near-field pairwise interactions dramatically decreased to $64 \times 27 \times 24^{2}=995,328$ and $512 \times 27 \times 3^{2}=124,416$, respectively. Computational time in the CMM program is the sum of three terms: time for (a) near-field, (b) far-field, and (c) remaining $\infty-27$ cell interactions. The first term (a) depends on the average number of atoms $\mathrm{N}$ in the deepest cell and is proportional to $\mathrm{O}\left(\mathrm{N}^{2}\right)$. The more atoms are in the deepest cell, the more computational time it will take. Computational time for the second term (b) is approximately linear with $\mathrm{O}(\mathrm{K})$, where $\mathrm{K}$ is the number of far cells. The last term (c) is constant at each level for a given system. Thus, there exists an optimal level at which a large number of interactions in the near-field will be compensated by relatively fewer interactions in the far-field. Compared with the Ewald summation method, the error in total energy at each level for CMM is less than $0.1 \%$, and the relative root mean square (RMS) of force is about 0.045 . The level has little influence on the accuracy for this case.

Regarding the simple and complex downward algorithms in the Taylor expansion, the latter is faster than the simple downward while maintaining the same order of errors in potential energy and RMS force. In the complex downward procedure, the Taylor series is obtained at the center of a parent cell. Then, it is shifted to the center of each children cell by multiplying the distance between the centers of the parent cell and its children cell. Starting at the level-1 cells and recursively repeating this procedure, we can compute the Taylor coefficients $\mathrm{V}^{(\mathbf{i})}$ for all the cells at all levels. The complex downward expands the Taylor series to each children cell only once instead of eight times in the simple downward. It can be seen in Figure 1 that at level 3, computational time is improved by $26 \%$ while accuracy remains the same. At level 2 , there is no apparent 
difference in CPU time and accuracy between the simple and complex downward algorithms since there are a large number of near-field interactions determining computational time and accuracy.

We also have studied dendrimers, water-methane binary mixtures, as well as watermethane-dendrimer ternary mixtures with various compositions. The optimal results for bulk systems are given in Table 4. Results show that the level of CMM (or average atom occupancy) is a key parameter in determining computational time and accuracy. The optimal value for average atom occupancy in the leaf cell is $3 \sim 10$ atoms. Under the optimal condition, the complex downward algorithm was preferred since it is generally faster than the simple downward method without losing its accuracy.

\section{B. MD simulations of confined systems}

PAMAM dendrimer has an ammonia core and $-\mathrm{CH}_{2} \mathrm{CH}_{2} \mathrm{CONHCH}_{2} \mathrm{CH}_{2} \mathrm{NH}_{2}$ monomer units. Additional layers of monomer units can attach to the nitrogen atoms of monomers such that the dendrimer grows like a tree (in contrast to single chain polymer). PAMAMs of generation 3 (G-3), 4, 5, and 6 have 382, 814, 1678, and 3406 atoms, respectively. Nonbond interactions come from both inter- and intra-molecular contributions, excluding 1-2 and 1-3 interactions. PAMAM dendrimers were built using POLYGRAF from Professor William A. Goddard III at California Institute of Technology. The universal force field (UFF) was used to obtain LJ parameters, and the charge equilibration ( $\mathrm{QEq}$ ) method was applied to assign charge to each atom in PAMAM dendrimers. In this work, the EW3D as applied to quasi-2D systems was used to check CMM2D results. Figure 2 shows that van der Waals and Coulomb potential energies converge within a certain range of vacuum height in the $\mathrm{z}$ direction using the Ewald summation technique. ${ }^{[20,21]}$ According to Spohr ${ }^{[21]}$, the conventional EW3D technique with an appropriate vacuum space in the $\mathrm{z}$ direction reproduced EW2D results although it is still quite time consuming as compared to CMM2D.

As shown in Figure 3, computational time decreases dramatically as the level increases for $\mathrm{N}>1000$. For example, for a G-5 dendrimer having 1678 atoms, as compared with level 1, computational time decreases by 4.4 and 6.2 times at levels 2 and 
3, respectively. For a G-6 dendrimer having 3406 atoms, computational time decreases by 4.1 and 10.7 times at levels 2 and 3, respectively. Thus, the larger the system is, the more computationally efficient the CMM method is. For large systems computational time is approximately linear with number of atoms. However, since there are a large number of atoms in leaf cells at level 1, the near-field interactions dominate overall computations. Thus, computational time depends quadratically $\left(\mathrm{N}^{2}\right)$ on number of atoms. Comparing the accuracy of CMM with that of the Ewald summation, the error in energy at levels 2 and 3 is comparable and is less than 1\% for various systems. RMS force is about 0.012 . Relatively large error in energy was observed at level 1 . The optimal value for average atom occupancy is 3 10 atoms at the deepest level. The complex downward algorithm is generally faster than the simple one without losing its accuracy.

Furthermore, we applied our CMM2D method to a more complex ternary system containing one G-4 PAMAM dendrimer, fourteen water and seven methane molecules. For all LJ cross-term parameters, the geometric mean combing rule was used, namely, $\varepsilon_{i j}=\sqrt{\varepsilon_{i j} \cdot \varepsilon_{i j}}$ and $\sigma_{i j}=\sqrt{\sigma_{i i} \cdot \sigma_{i j}}$. Figure 4 illustrates that the EW3D simulation does produce a converged region beyond $200 \AA$ in the $\mathrm{z}$ direction for van der Waals and Coulombic potential energies. Figure 5 shows that potential energy and RMS force at the level 1 are more accurate than those at levels 2 and 3. However computational time at level 1 significantly increases when compared with that at levels 2 and 3. As shown in Table 6, an optimal result with accurate potential energy and RMS force, as well as fast computational time can be achieved at level 2. Final optimal results for pure components (water or dendrimer), water-methane binary mixtures, and water-methane-dendrimer ternary systems are listed in Table 7 , in which the optimal level (or average atom occupancy) and the complex downward algorithm are shown. The percentage error in total energy is about $1 \%$ and the RMS force is about 0.014 .

\section{GCMC simulations of confined systems}

A system with a dimension of $46.7 \times 59.6 \times 57.3 \AA$ was tested for a binary mixture of G-5 PAMAM dendrimer and water in a confined system using our CMM-GCMC program. The difference in energy $\triangle \mathrm{E}$ between two different configurations was 
calculated at each level for each GCMC move. Results in Table 8 show that potential energies calculated from our CMM-GCMC program for various GCMC moves are consistent with those from our CMM-MD program. The slight difference among different levels for each move is due to approximations used in the calculation of far-field contributions.

\section{CONCLUSIONS}

The CMM method is efficient in calculating both van der Waals and Coulombic interactions with significantly low computational time and good accuracy. It is well suited to handling large systems with long-range interactions in molecular simulations. Simulation results show that the level of the cell hierarchy tree (or average atom occupancy) in the deepest cell is a key factor in determining computational time and accuracy. The optimal number of atoms in the deepest cell is $3 \sim 10$. The complex Taylor expansion algorithm is faster than the simple one without losing its accuracy. Under the optimal conditions, average error in total potential energy is less than $1 \%$ and RMS force is about 0.015 when compared with the Ewald summation method while computational efficiency improves significantly. Results also show that computational time of the CMM method scales almost linearly with the number of atoms in the cell for large systems.

The CMM method was extended to confined systems from bulk systems in MD simulations. Potential energies calculated from our CMM2D program are consistent with those from the EW3D technique applied to quasi-2D systems by adding a sufficiently large vacuum space on the top of the slab. The difference in total potential energy between these two methods is $\sim 1 \%$. Moreover, the CMM method was applied to GCMC simulations for both $3 \mathrm{D}$ and $2 \mathrm{D}$ systems. Our CMM code is readily incorporated in the GCMC, MD, and grand canonical molecule simulation (GCMD) programs for applications, such as studies of adsorption and diffusion in pores.

\section{ACKNOWLEDGMENTS}

We gratefully acknowledge the Department of Energy for financial support. 


\section{REFERENCES:}

[1]. K.-T. Lim, et al., J. Comput. Chem., 18, 501 (1997)

[2]. H.-Q. Ding, N. Karasawa, and W.A. Goddard III, J. Chem. Phys., 97, 4309 (1992)

[3]. H.-Q. Ding, N. Karasawa, and W.A. Goddard III, Chem. Phys. Lett., 196, 6 (1992)

[4]. U. Essmann, L. Perera, M.L. Berkowitz, T. Darden, H. Lee, and L.G. Pedersen, J. Chem. Phys. 103, 8577 (1995)

[5]. A.Y. Toukmaji and J.A. Board Jr., Comput. Phys. Commun. 95, 73 (1996)

[6]. Appel, A.W. SIAM J. Sci. Statist. Comput., 6, 85 (1985)

[7]. J. Carrier, L. Greengard, Rokhlin, V. SIAM J. Sci. Statist. Comput., 9, 669 (1989)

[8]. P. Vashishta, et al., Materi. Sci. Eng., B37, 56 (1996)

[9]. D.E.Parry, Surf. Sci., 49, 433 (1975); 54, 195 (1976)

[10]. D.M. Heyes, M. Barber, and J.H.R. Clarke, J. Chem. Soc., Faraday Trans. II 73, 1485 (1977)

[11]. D.M. Heyes, J. Phys. Chem., Solids 41, 291 (1980)

[12]. D.M. Heyes and F. van Swol, J. Chem. Phys., 75, 5051 (1981)

[13]. D.M. Heyes, J. Chem. Phys., 79, 4010 (1983)

[14]. D.M. Heyes, Phys. Rev. B, 30, 2182 (1984)

[15]. D.M. Heyes, Surf. Sci., 110, 1619 (1981)

[16]. D.M. Heyes, Condensed Matter, 49, II, 755 (1994)

[17]. J.Lekner, Physica., A 176, 485 (1991)

[18]. J.Hautman and M.L. Klein, Mol. Phys., 75, 379 (1992)

[19]. S.W. de Leeuw, J.W. Perram, and E.R. Smith, Proc. R. Soc., London Ser. A373, 27 (1980)

[20]. Jiang, S.; Frazier, R.; Yamaguchi, E.S.; Blanco, M.; Dasgupta, S.; Zhou, Y.; Cagin, T.; Tang, Y.; Goddard, W.A., III J. Phys. Chem. B 101, 7702 (1997)

[21]. E. Spohr, J. Chem. Phys., 107, 6342 (1997)

[22]. S.Y. Liem and J.H.R Clarke, Mol. Phys., 92, 19 (1997)

[23]. A. Grzybowski, E. Gwóźdź, and A.Bródka, Phys. Rev. B., 61, 6706 (2000-II)

[24]. In-Chul Yeh and M. L. Berkowitz, J. Chem. Phys., 111, 3155 (1999) 
Table 1. Computation of energy, RMS force, and CPU time for 512 water molecules in the bulk system from the Ewald summation, MPSim, minimum image, and our CMM3D program.

\begin{tabular}{|c|c|c|c|c|c|c|c|}
\hline Method & $\begin{array}{c}\text { Taylor } \\
\text { expansion }\end{array}$ & Level & $M^{b}$ & $\mathrm{~N}^{c}$ & $\begin{array}{c}\text { Energy }^{d} \\
(\mathrm{kcal} / \mathrm{mol})\end{array}$ & RMS force & $\begin{array}{c}\text { Time } \\
(s)\end{array}$ \\
\hline Ewald & & & & & $(-1343.47)$ & & \\
\hline Min. image & & & & & -49565.55 & & \\
\hline MPSim & & 1 & 8 & 192 & -2.127 & 0.0395 & \\
\hline \multirow[t]{2}{*}{$\mathrm{CMM}$} & Simple & 1 & 8 & 192 & 0.737 & 0.0395 & 132 \\
\hline & Complex $^{a}$ & 1 & I & I & I & I & I \\
\hline MPSim & & 2 & 64 & 24 & -1.994 & 0.0449 & \\
\hline CMM & Simple & 2 & 64 & 24 & 0.866 & 0.0366 & 21 \\
\hline CMM & Complex ${ }^{a}$ & 2 & 64 & 24 & 0.870 & 0.0449 & 21 \\
\hline MPSim & & 3 & 512 & 3 & -1.600 & 0.0449 & \\
\hline CMM & Simple & 3 & 512 & 3 & 1.263 & 0.0292 & 31 \\
\hline CMM & Complex ${ }^{a}$ & 3 & 512 & 3 & 1.270 & 0.0449 & 23 \\
\hline
\end{tabular}

a. Simple or complex downward algorithm in the Taylor expansion described in Section 2 and no complex downward for level 1.

b. Number of cells $M$ in the deepest level.

c. Average number of atoms $n$ in the leaf cell.

d. The potential energy is given for the Ewald summation in parentheses. Only the differences are given for other methods.

e. $r m s=\left(\frac{\sum_{i=1}^{N}\left|F_{i}^{\text {CMM IMPSim }}-F_{i}^{E \text { wald }}\right|^{2}}{\sum_{i=1}^{N}\left|F_{i}^{\text {Ewald }}\right|^{2}}\right)^{1 / 2}$ 
Table 2. Energy decompositions for van der Waals and Coulombic interactions at each level for 512 water molecules in the bulk from our CMM3D program.

\begin{tabular}{|c|c|c|c|c|c|c|}
\hline \multirow[t]{2}{*}{$\begin{array}{c}\text { Taylor } \\
\text { expansion }\end{array}$} & \multirow[t]{2}{*}{ Level } & \multirow[t]{2}{*}{$\mathbf{n}$} & \multicolumn{2}{|c|}{$\begin{array}{l}\text { van der Waals Sum } \\
\qquad(\mathrm{kcal} / \mathrm{mol})\end{array}$} & \multicolumn{2}{|c|}{$\begin{array}{l}\text { Electrostatic Sum } \\
\qquad(\mathrm{kcal} / \mathrm{mol})\end{array}$} \\
\hline & & & Dispersion & Repulsion & CMM & RCMM \\
\hline Simple & 1 & 192 & -257.963 & 22.361 & -101.404 & -1005.728 \\
\hline Simple & 2 & 24 & -257.916 & 22.361 & -101.321 & -1005.728 \\
\hline Complex & 2 & 24 & -257.907 & 22.361 & -101.327 & -1005.728 \\
\hline Simple & 3 & 3 & -257.935 & 22.361 & -100.905 & -1005.728 \\
\hline Complex & 3 & 3 & -257.900 & 22.361 & -100.932 & -1005.728 \\
\hline
\end{tabular}


Table 3. Energy from near- and far-field contributions for van der Waals and Coulombic interactions on each level for 512 water molecules in the bulk system from our CMM3D program.

\begin{tabular}{|c|c|c|c|c|c|c|c|c|c|c|}
\hline \multirow[t]{2}{*}{$\begin{array}{c}\text { Taylor } \\
\text { expansion }\end{array}$} & \multirow[t]{2}{*}{$\begin{array}{c}\text { Cutoff } \\
\text { (A) }\end{array}$} & \multirow[t]{2}{*}{ Level } & \multirow[t]{2}{*}{$\mathbf{n}$} & \multicolumn{2}{|c|}{$\begin{array}{c}\text { van der Waals } \\
\text { dispersion } \\
(\mathrm{kcal} / \mathrm{mol}) \\
\end{array}$} & \multicolumn{2}{|c|}{$\begin{array}{c}\text { van der Waals } \\
\text { repulsion } \\
(\mathrm{kcal} / \mathrm{mol}) \\
\end{array}$} & \multicolumn{3}{|c|}{$\begin{array}{l}\text { Coulomb } \\
(\mathrm{kcal} / \mathrm{mol})\end{array}$} \\
\hline & & & & near & far & near & far & near & far & $R C M M$ \\
\hline Simple & 27 & 1 & 192 & -257.651 & -0.311 & 22.361 & $1.21 \mathrm{E}-7$ & -101.259 & -0.154 & -1005.728 \\
\hline Simple & 13.5 & 2 & 24 & -255.285 & -2.631 & 22.361 & $4.98 \mathrm{E}-5$ & -100.554 & -0.767 & -1005.728 \\
\hline Complex & 13.5 & 2 & 24 & -255.285 & -2.622 & 22.361 & 4.98E-5 & -100.554 & -0.772 & -1005.728 \\
\hline Simple & 6.75 & 3 & 3 & -241.712 & -16.223 & 22.354 & 7.47E-3 & -97.688 & -3.217 & -1005.728 \\
\hline Complex & 6.75 & 3 & 3 & -241.712 & -16.188 & 22.354 & $7.47 \mathrm{E}-3$ & -97.688 & -3.244 & -1005.728 \\
\hline
\end{tabular}


Table 4. CPU time, RMS force, and relative energy error for bulk systems with the optimal parameter $\mathrm{n}$ and using the complex downward algorithm.

\begin{tabular}{ccccccc}
\hline System & $\mathrm{N}$ & Level & $\mathrm{n}$ & $\begin{array}{c}\text { Energy Error } \\
(\%)\end{array}$ & $\begin{array}{c}\text { RMS } \\
\text { force }\end{array}$ & $\begin{array}{c}\text { Time } \\
(s)\end{array}$ \\
\hline PURE & & & & & & \\
water & 512 & 3 & 3 & -0.09 & 0.0449 & 23 \\
dendrimer & 382 & 2 & 6 & -0.281 & 0.0214 & 2 \\
& 814 & 2 & 13 & -0.109 & 0.0067 & 14 \\
& 1678 & 3 & 3.3 & -0.676 & 0.0087 & 39 \\
& 3406 & 3 & 7 & 0.931 & 0.0066 & 87 \\
\hline BINARY MIXTURES & & & & & & \\
\hline water-methane & 462 & 2 & 7.2 & -1.056 & 0.0022 & 7 \\
& 609 & 2 & 9.5 & 0.889 & 0.0187 & 4 \\
& 1848 & 3 & 3.6 & 0.682 & 0.0173 & 20 \\
& 3440 & 3 & 6.7 & 0.881 & 0.0433 & 39 \\
\hline TERNARY MIXTURES & & & & & & \\
\hline dendrimer-water-methane & 422 & 2 & 6.6 & -0.741 & 0.0008 & 6 \\
& 891 & 2 & 14 & -0.845 & 0.0009 & 18 \\
\hline
\end{tabular}


Table 5. Computation of energy and CPU time for one G-3, 4, 5, or 6 PAMAM dendrimer in the confined system from the Ewald sum and our CMM2D program.

\begin{tabular}{|c|c|c|c|c|c|c|c|c|}
\hline $\mathbf{N}$ & Method & $\begin{array}{c}\text { Taylor } \\
\text { expansion }\end{array}$ & Level & $\mathbf{M}$ & $\mathrm{n}$ & $\begin{array}{c}\text { Energy } \\
(\mathrm{kcal} / \mathrm{mol})\end{array}$ & $\begin{array}{l}\text { RMS } \\
\text { force }\end{array}$ & $\begin{array}{c}\text { Time } \\
(s)\end{array}$ \\
\hline \multirow[t]{7}{*}{382} & Ewald & & & & & $(-14143.94)$ & & \\
\hline & CMM & Simple & 1 & 8 & 48 & 146.91 & 0.01188 & 4 \\
\hline & CMM & Complex & 1 & I & 1 & / & I & 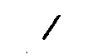 \\
\hline & CMM & Simple & 2 & 64 & 6 & 55.12 & 0.02032 & 2 \\
\hline & $\mathrm{CMM}$ & Complex & 2 & 64 & 6 & 35.00 & 0.02066 & 1 \\
\hline & $\mathrm{CMM}$ & Simple & 3 & 512 & 0.7 & 144.11 & 0.05081 & 15 \\
\hline & CMM & Complex & 3 & 512 & 0.7 & 139.27 & 0.05204 & 11 \\
\hline \multirow[t]{7}{*}{814} & Ewald & & & & & $(-558.880)$ & & \\
\hline & CMM & Simple & 1 & 8 & 102 & -2.405 & 0.00166 & 25 \\
\hline & CMM & Complex & 1 & I & I & I & I & $/$ \\
\hline & $\mathrm{CMM}$ & Simple & 2 & 64 & 12.7 & 0.745 & 0.00504 & 7 \\
\hline & CMM & Complex & 2 & 64 & 12.7 & 0.737 & 0.00510 & 7 \\
\hline & $\mathrm{CMM}$ & Simple & 3 & 512 & 1.6 & 1.711 & 0.01222 & 18 \\
\hline & CMM & Complex & 3 & 512 & 1.6 & 1.892 & 0.01318 & 14 \\
\hline \multirow[t]{7}{*}{1678} & Ewald & & & & & (235.939) & & \\
\hline & $\mathrm{CMM}$ & Simple & 1 & 8 & 210 & 6.241 & 0.00221 & 124 \\
\hline & CMM & Complex & 1 & 1 & I & 1 & 1 & I \\
\hline & $\mathrm{CMM}$ & Simple & 2 & 64 & 26 & -3.156 & 0.00328 & 29 \\
\hline & CMM & Complex & 2 & 64 & 26 & -4.659 & 0.00353 & 28 \\
\hline & CMM & Simple & 3 & 512 & 3.3 & -2.206 & 0.00851 & 25 \\
\hline & CMM & Complex & 3 & 512 & 3.3 & -3.256 & 0.00869 & 20 \\
\hline \multirow[t]{7}{*}{3406} & Ewald & & & & & $(168.237)$ & & \\
\hline & CMM & Simple & 1 & 8 & 428 & 1.319 & 0.00155 & 526 \\
\hline & CMM & Complex & 1 & I & I & 1 & 1 & I \\
\hline & $\mathrm{CMM}$ & Simple & 2 & 64 & 63 & -3.531 & 0.00257 & 128 \\
\hline & CMM & Complex & 2 & 64 & 63 & -3.921 & 0.00258 & 128 \\
\hline & $\mathrm{CMM}$ & Simple & 3 & 512 & 6.7 & -4.000 & 0.00641 & 54 \\
\hline & $\mathrm{CMM}$ & Complex & 3 & 512 & 6.7 & -3.939 & 0.00658 & 49 \\
\hline
\end{tabular}


Table 6. Computation of energy, RMS force, and CPU time for one G-4 PAMAM dendrimer, fourteen water, and seven methane molecules in the confined system from the Ewald summation and our CMM2D program.

\begin{tabular}{ccccccccc}
\hline $\mathrm{N}$ & Method & $\begin{array}{c}\text { Taylor } \\
\text { expansion }\end{array}$ & Level & $\mathrm{M}$ & $\mathrm{n}$ & $\begin{array}{c}\text { Energy } \\
(\mathrm{kcal} / \mathrm{mol})\end{array}$ & $\begin{array}{c}\text { RMS } \\
\text { force }\end{array}$ & $\begin{array}{c}\text { Time } \\
(s)\end{array}$ \\
\hline 891 & Ewald & & & & & $(-592.50)$ & & \\
& $\mathrm{CMM}$ & Simple & 1 & 8 & 111.3 & -0.530 & 0.00183 & 38 \\
& $\mathrm{CMM}$ & Complex & 1 & $/$ & $/$ & $/$ & $/$ & $/$ \\
& $\mathrm{CMM}$ & Simple & 2 & 64 & 13.9 & 2.244 & 0.00638 & 9 \\
& $\mathrm{CMM}$ & Complex & 2 & 64 & 13.9 & 2.078 & 0.00641 & 9 \\
& $\mathrm{CMM}$ & Simple & 3 & 512 & 1.7 & 4.091 & 0.01418 & 19 \\
$\mathrm{CMM}$ & Complex & 3 & 512 & 1.7 & 4.901 & 0.01522 & 15 \\
\hline
\end{tabular}


Table 7. CPU time and percentage error in potential energy with an optimum level and using the complex downward algorithm for the pure (water and PAMAM dendrimer), binary (water, methane) and ternary (water, methane, and dendrimer) systems from our CMM2D program.

\begin{tabular}{lccccccc}
\hline \multicolumn{1}{c}{ System } & N & Level & M & n & $\begin{array}{c}\text { Error } \\
(\%)\end{array}$ & $\begin{array}{c}\text { RMS } \\
\text { Force }\end{array}$ & $\begin{array}{c}\text { Time } \\
(s)\end{array}$ \\
\hline PURE & & & & & & & \\
water & 1536 & 3 & 512 & 3 & -0.136 & 0.0483 & 16 \\
dendrimer & 382 & 2 & 64 & 6 & -0.247 & 0.0207 & 2 \\
& 814 & 2 & 64 & 12.7 & -0.132 & 0.0051 & 7 \\
& 1678 & 3 & 512 & 3.3 & -1.380 & 0.0087 & 20 \\
& 3406 & 3 & 512 & 6.7 & -2.341 & 0.0066 & 49 \\
\hline BINARY MIXTURES & & & & & & & \\
\cline { 1 - 2 } & 462 & 2 & 64 & 7.2 & -0.315 & 0.0054 & 2 \\
& 609 & 2 & 64 & 9.5 & 0.921 & 0.0160 & 2 \\
& 1848 & 3 & 512 & 3.6 & 0.551 & 0.0160 & 18 \\
& 2208 & 3 & 512 & 4.3 & -1.990 & 0.0164 & 20 \\
\hline TERNARY MLXTURES & & & & & & & \\
dendrimer-water-methane & 422 & 2 & 64 & 6.6 & -0.972 & 0.0008 & 2 \\
& 891 & 2 & 64 & 13.9 & -0.351 & 0.00641 & 9 \\
\hline
\end{tabular}


Table 8. Comparison of potential energy from our CMM-MD and CMMGCMC programs.

\begin{tabular}{|c|c|c|c|c|c|}
\hline \multirow[b]{2}{*}{$A D D$} & \multirow[b]{3}{*}{ simple } & \multicolumn{3}{|c|}{ CMM-MD } & \multirow{2}{*}{ 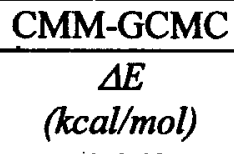 } \\
\hline & & $\begin{array}{c}\text { Old } \\
(\mathrm{kcal} / \mathrm{mol})\end{array}$ & $\begin{array}{c}\text { New } \\
(\mathrm{kcal} / \mathrm{mol})\end{array}$ & $\begin{array}{c}\Delta E \\
(\mathrm{kcal} / \mathrm{mol})\end{array}$ & \\
\hline level 1 & & 242.18 & 242.108 & -0.072 & -0.068 \\
\hline \multirow[t]{2}{*}{ level 2} & simple & 232.783 & 232.791 & 0.008 & 0.004 \\
\hline & complex & 231.28 & 231.293 & 0.013 & 0.017 \\
\hline \multirow[t]{2}{*}{ level 3} & simple & 233.733 & 233.717 & -0.016 & -0.025 \\
\hline & complex & 232.683 & 232.712 & 0.029 & 0.033 \\
\hline \multirow{2}{*}{\multicolumn{2}{|c|}{ DELETE }} & \multicolumn{3}{|c|}{ CMM-MD } & CMM-GCMC \\
\hline & & $\begin{array}{c}\text { Old } \\
(\mathrm{kcal} / \mathrm{mol})\end{array}$ & $\begin{array}{c}\mathrm{New} \\
(\mathrm{kcal} / \mathrm{mol})\end{array}$ & $\begin{array}{c}\Delta E \\
(\mathrm{kcal} / \mathrm{mol})\end{array}$ & $\begin{array}{c}\Delta E \\
(\mathrm{kcal} / \mathrm{mol})\end{array}$ \\
\hline level 1 & simple & 242.108 & 242.181 & 0.072 & 0.074 \\
\hline \multirow[t]{2}{*}{ level 2} & simple & 232.791 & 232.783 & -0.008 & 0.0003 \\
\hline & complex & 231.293 & 231.28 & -0.013 & -0.012 \\
\hline \multirow[t]{2}{*}{ level 3} & simple & 233.717 & 233.733 & 0.016 & 0.029 \\
\hline & complex & 232.712 & 232.683 & -0.029 & -0.028 \\
\hline \multirow{2}{*}{\multicolumn{2}{|c|}{ MOVE }} & \multicolumn{3}{|c|}{ CMM-MD } & CMM-GCMC \\
\hline & & $\begin{array}{c}\text { Old } \\
(\mathrm{kcal} / \mathrm{mol})\end{array}$ & $\begin{array}{c}\text { New } \\
(\mathrm{kcal} / \mathrm{mol})\end{array}$ & $\begin{array}{c}\Delta E \\
(\mathrm{kcal} / \mathrm{mol})\end{array}$ & $\begin{array}{c}\Delta E \\
(k c a l / m o l)\end{array}$ \\
\hline level 1 & simple & 242.1079 & 242.4757 & 0.368 & 0.364 \\
\hline \multirow{2}{*}{ level 2} & simple & 232.7917 & 233.2285 & 0.437 & 0.433 \\
\hline & complex & 231.2931 & 231.7315 & 0.438 & 0.435 \\
\hline \multirow[t]{2}{*}{ level 3} & simple & 233.7171 & 234.1922 & 0.475 & 0.455 \\
\hline & complex & 232.7122 & 233.211 & 0.499 & 0.495 \\
\hline
\end{tabular}




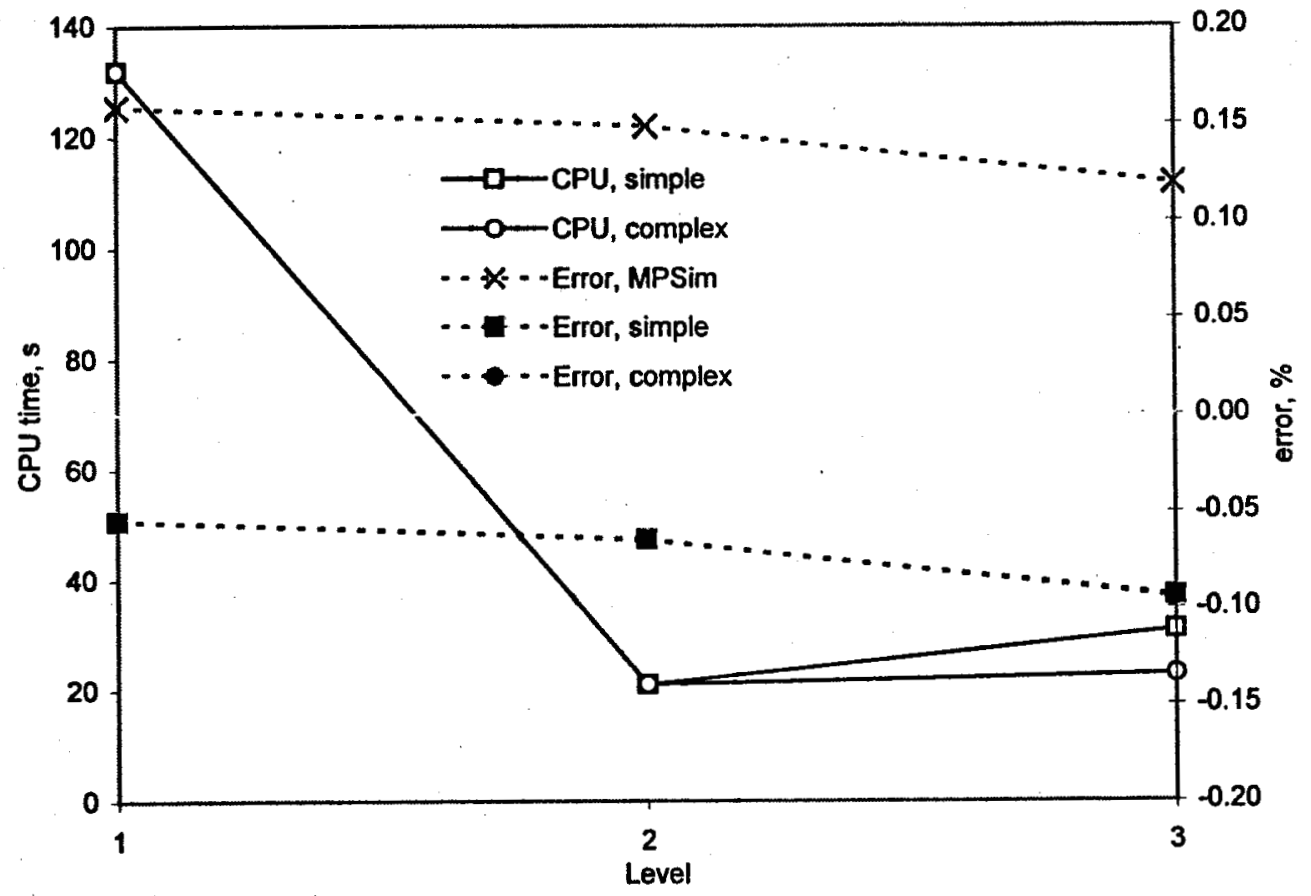

Figure 1. CPU time and percentage error in potential energy for 512 water molecules in the bulk from our CMM program. Three cell hierarchy trees were built (level 1,2,3) and two Taylor expansion algorithms (simple and complex downward) were implemented to evaluate multipoles in the far-field. 
(a)

G-3 PAMAM

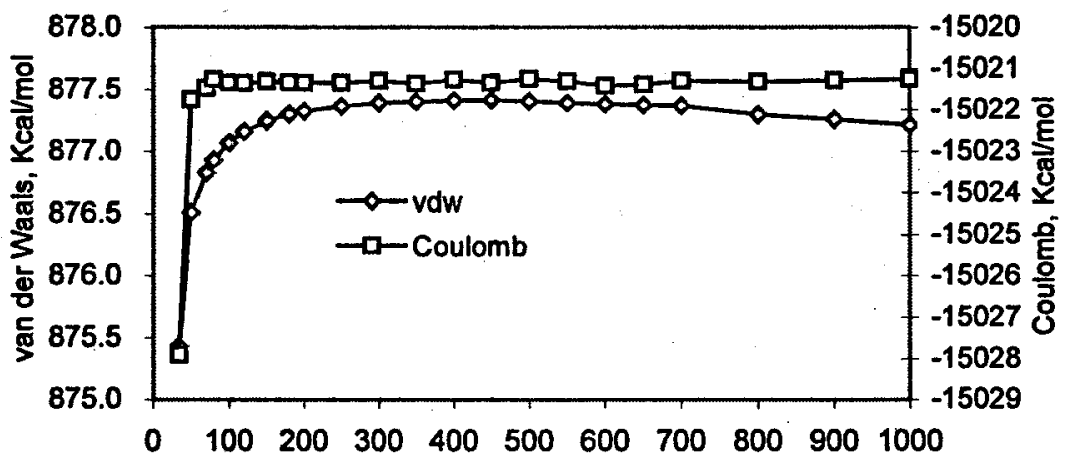

(b)

G-4 PAMAM

(c)

G-5 PAMAM

(d)

G-6 PAMAM
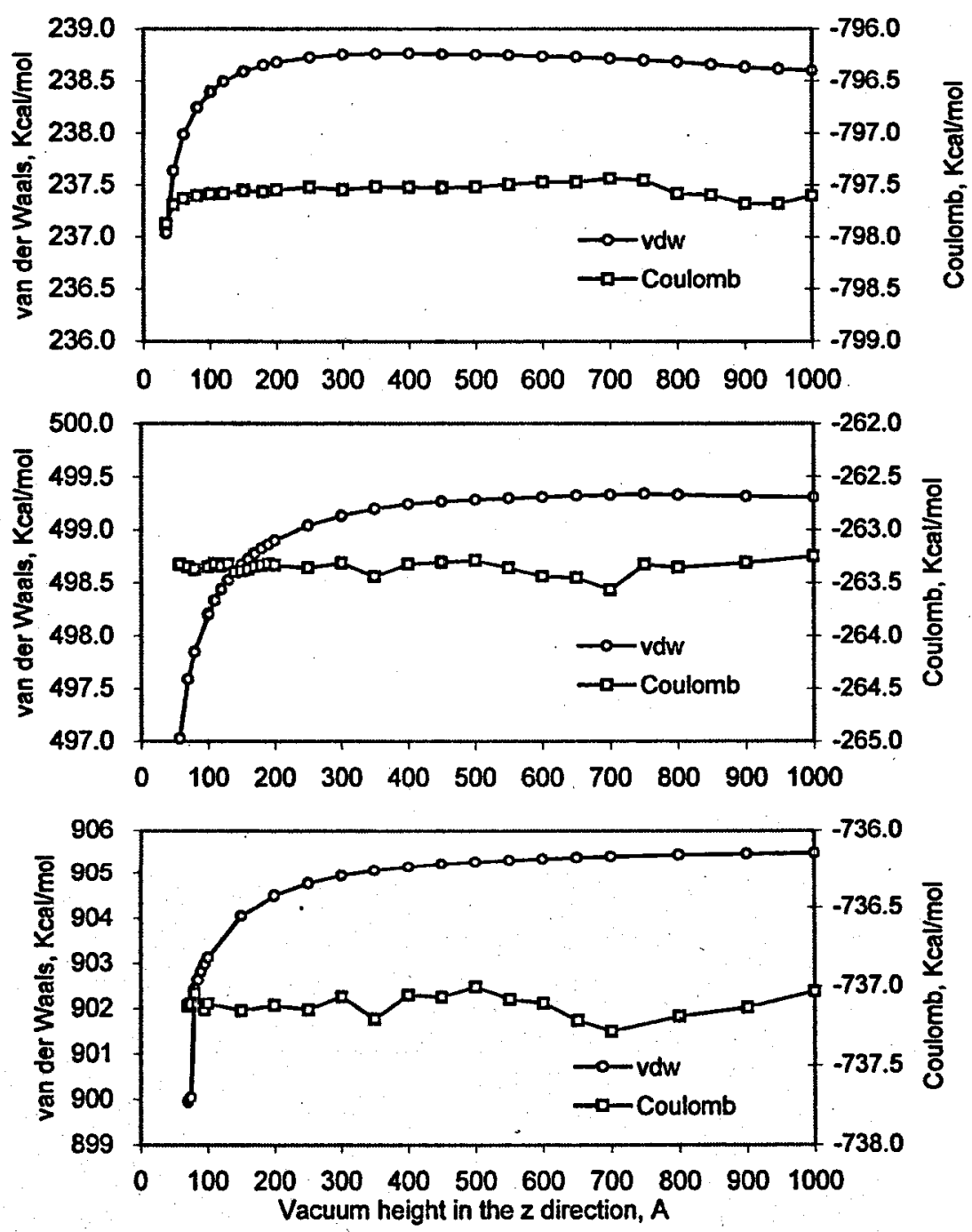

Figure 2. van der Waals and Coulombic potential energies vs vacuum height in the $z$ direction for PAMAM dendrimers in the confined system from the Ewald summation.

(a) $v d w=877.359$ and Coulomb $=-15021.303$ for G-3 PAMAM with 382 atoms

(b) $v d w=238.657$ and Coulomb $=-797.537$ for G-4 PAMAM with 814 atoms

(c) $v d w=499.290$ and Coulomb $=263.351$ for G-5 PAMAM with 1678 atoms

(d) $v d w=905.357$ and Coulomb $=-737.120$ for G-6 PAMAM with 3406 atoms 

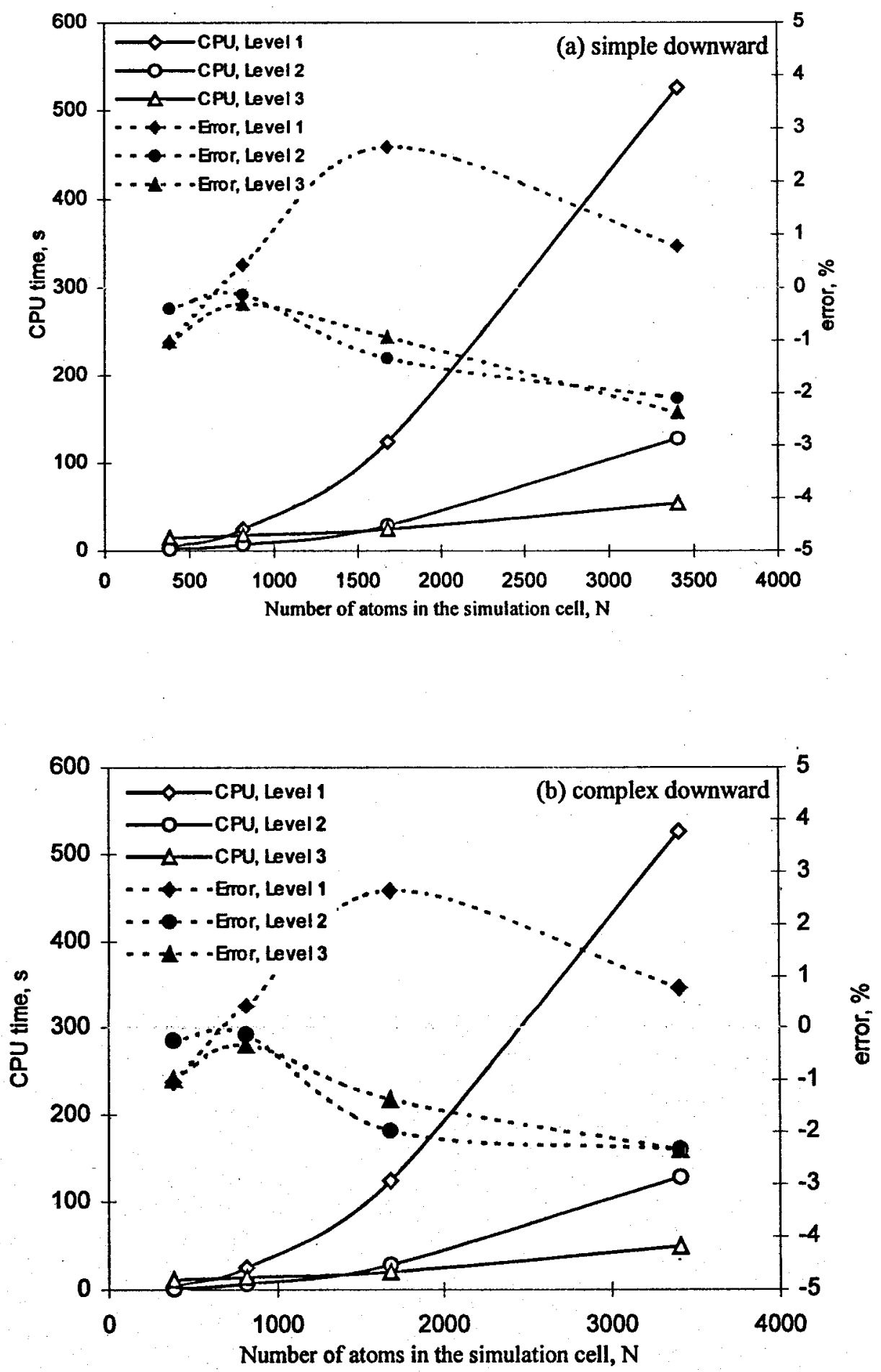

Figure 3. CPU time and percentage error in potential energy for PAMAM dendrimer molecules in the confined system from our CMM2D program. Three cell hierarchy trees were built (level 1, 2,3) and two Taylor expansion algorithms (simple and complex downward) were implemented to evaluate multipoles in the far-field. 


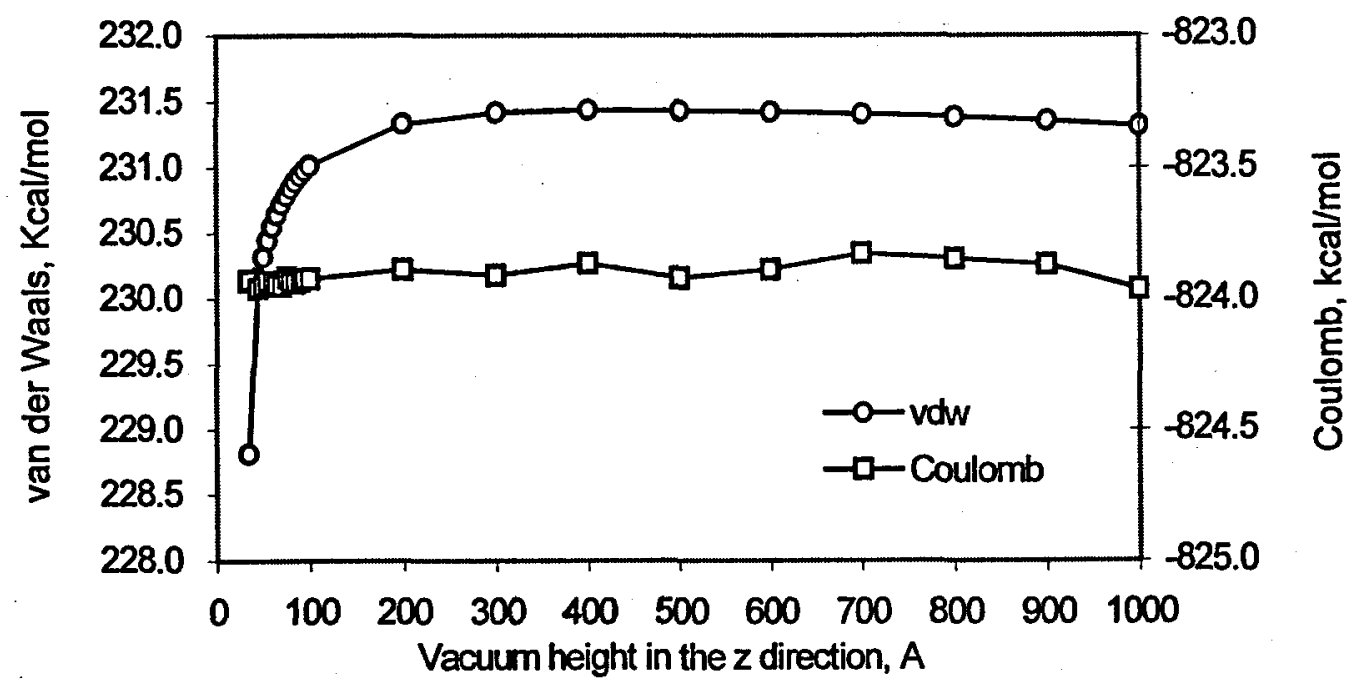

Figure 4. van der Waals and Coulombic potential energies vs vacuum height in the $\mathrm{z}$ direction for one G-4 PAMAM dendrimer, fourteen water and seven methane molecules in the confined system from the Ewald sum. vdw and Coulombic potential energies converge between 300 and $1000 \AA$. The average values of vdw and Coulomb are 231.387 and $-823.887 \mathrm{kcal} / \mathrm{mol}$, respectively. 


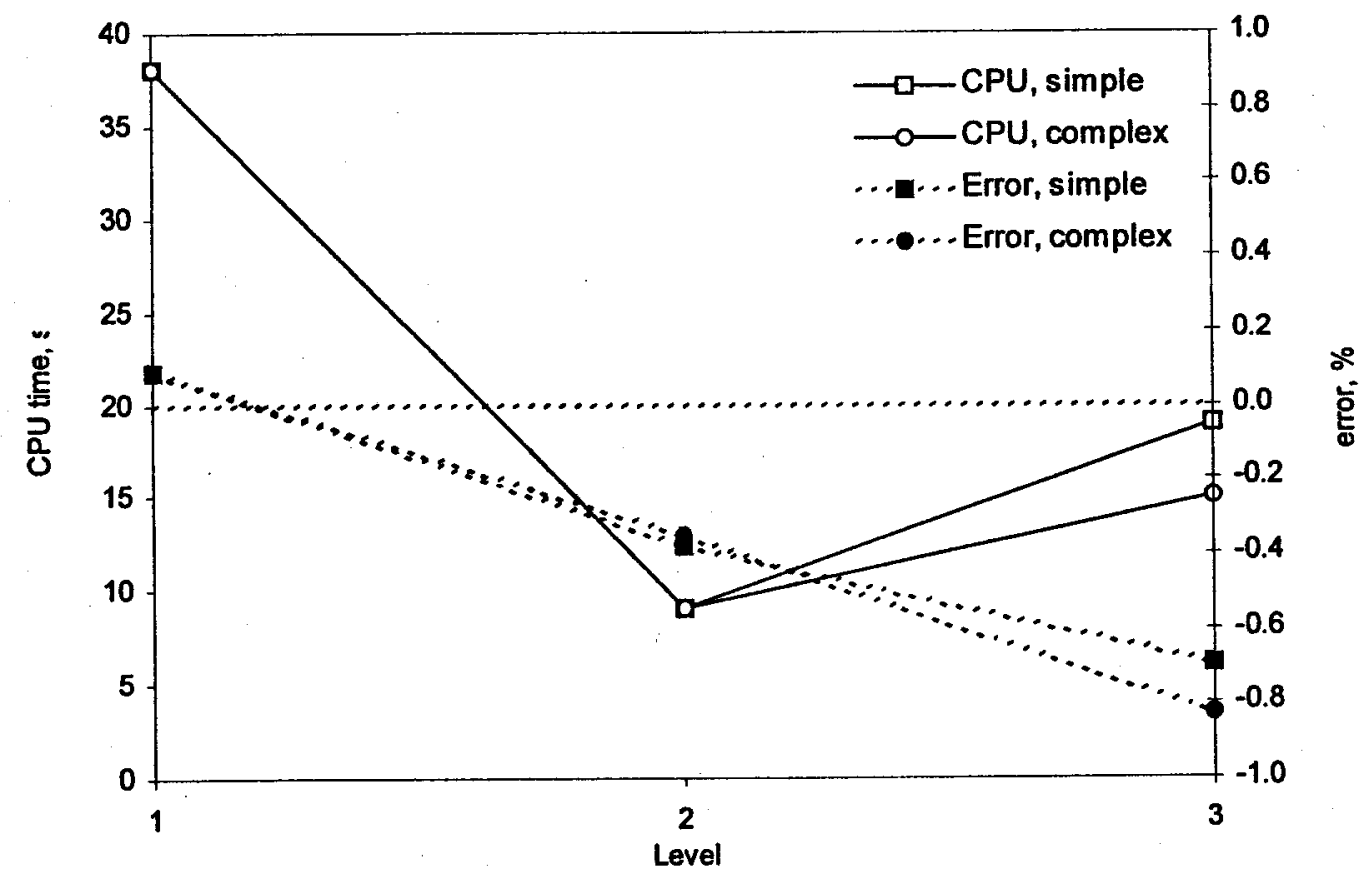

Figure 5. CPU time (solid line) and percentage error in potential energy (dash line) for one G-4 PAMAM dendrimer, fourteen water and seven methane molecules in the confined system from our CMM2D program. Three cell hierarchy trees were built (level 1,2,3) and two Taylor expansion algorithms (simple and complex downward) were implemented to evaluate multipoles in the far-field. 